\title{
An examination of concept lattices, types, and functions in Roget's International Thesaurus
}

\author{
JOHN BRADY \\ University of Arkansas at Little Rock \\ and Systematics Information Services, Inc., Little Rock, Arkansas
}

\begin{abstract}
The notions of objects and attributes are essential to knowledge representation. Rudolf Wille's concept lattices group objects and attributes into concepts, with concepts organized in a lattice, so that some concepts are subconcepts of others. This paper applies certain aspects of type theory to concept lattices in order better to facilitate the representation of knowledge in Roget's International Thesaurus. Of particular interest is a formal analysis of functions providing concept lattices with a way of representing procedures and actions that currently are not present in the thesaurus.
\end{abstract}

The notions of objects and attributes are essential to knowledge representation, as well as to object-oriented programming. Wille's (1990) concept lattices group objects and attributes into concepts, with concepts organized in a lattice, so that some concepts are subconcepts of others. Cardelli and Wegner (1985) have examined objects and attributes in terms of types, as they advance the understanding of types into object-oriented programming. My objective is to apply certain aspects of the type to concept lattices in order better to facilitate the representation of knowledge in Roget's International Thesaurus (1962; hereafter referred to as R.I.T.). Of particular interest are those aspects of the type involving functions, quantification, parametric types, polymorphism, and data abstraction. This paper will focus on the application of functions to concept lattices and to R.I.T. A formal analysis of functions in concept lattices will provide a way to represent procedures and actions that currently are not present in the thesaurus. For example, Category 329 mentions words about cooking, kitchens, utensils, and manners of cooking. However, nowhere in that category is there a function for making toast (e.g., MAKE-TOAST: ELECTRICTOASTER $\times$ BREAD $\rightarrow$ TOAST). Not only will the application of functions to concept lattices allow procedures and actions to be represented, but functions also will show relations between concepts that may appear in divergent points of the lattice. To achieve this objective, I will first show how a concept lattice of $R . I . T$. is represented in object-oriented terms. I will follow that up with a more formalized analysis using Cardelli and Wegner's notion of the type.

\section{Statement of Problem}

R.I.T. is structured into a semantic hierarchy. The top layer of the hierarchy is composed of eight general classes. Those classes are divided and subdivided again until the layer consisting of 1,040 categories is reached. Each of

Correspondence should be addressed to J. Brady, 4 DeGray Cove, Maumelle, AR 72113. the 1,040 categories is divided into $10-15$ paragraphs, with each paragraph grouped into semicolon groups of related words. An example of this hierarchy is given below:

\section{Class Three: Physics \\ II. Heat 329. \\ Cooking \\ .4 cook, do, prepare; boil, stew, sim- mer, parboil; brew; poach, coddle; bake, fire, oven-bake; scallop, es- callop; shirr; roast; toast; fry, griddle, pan; sauté; frizz, frizzle; sear, braise, brown; broil, grill, pan-broil; barbecue; fricassee; steam; devil; curry; baste, jipper; do to a turn, do to rags.}

One of the subdivisions of the Physics class is Heat. One of the subdivisions of Heat is the category Cooking. One of the paragraphs of Cooking is paragraph 4. Paragraph 329.4 (Category 329, paragraph 4) contains 21 semicolon groups of related words.

Even though R.I.T. is structured hierarchically, that hierarchical structure was not derived using any formal methodology. Moreover, the hierarchical structure inherent in R.I.T. is not facilitative for indicating the polysemous aspects of words. In order to produce a more formal hierarchical structure for the words in R.I.T., projects are underway at University of Arkansas at Little Rock under the direction of Sally Sedelow, Walter Sedelow, and Robert Kent to derive systematically a hierarchy using Wille's (1990) formal concept analysis. Using formal concept analysis, R.I.T. may be analyzed at the paragraph or semicolon group level to generate a concept lattice that shows related areas of R.I.T. The concept lattice will provide us with inheritance links that show how pairs of concepts are related.

A concept lattice is a very good way to represent the knowledge embedded within R.I.T. However, the one no- 
tion that appears to be missing from R.I.T., as well as from concept lattices, is the notion of representing procedures and functions that act on concepts. Not only will the application of functions to concept lattices allow procedures and actions to be represented, but functions also will show relations between concepts that may appear in divergent points of the lattice.

\section{Concept Lattices}

The concept lattice containing electric toaster, bread, and toast is represented in Figure 1 (Wille, 1990). The concept lattice shows the generators for each concept. As the lines in the concept lattice are traced from top to bottom, attributes are added as new attribute generators are encountered. As the lines are traced from bottom to top, new objects are added as new object generators are encountered. For example, the concept node labeled B16 contains the attributes \{bread, meat, provision\}, and contains the objects $\{306.15,306.1,783.3\}$.

It may be seen from the concept lattice that the concepts for toaster, bread, and toast are represented by separate concepts. The concept containing toaster is labeled B4, the concept containing bread is labeled B16, the concept containing the noun toast is labeled B10, and the concept containing the verb toast is labeled B3. Because the concept lattice shows inheritance links only, there is nothing to represent a link between these related concepts. Not only are these concepts not linked in the concept lattice, but the concepts are not linked in R.I.T. in any apparent way.

Besides the inheritance links provided by Wille's concept lattices, my research additionally should allow us to represent R.I.T. using other types of links that connect two or more concepts together. Thomason and Touretsky (1991) have developed an inheritance theory for knowledge representation that allows individuals and kinds of individuals to be linked together. Many types of links are available in their system, including IS-A links, IS-NOT-A links, relational links, roles, and so on (Thomason \& Touretsky, 1991, p. 237). In their system, there is no mathematical difference between an IS-A (inheritance link) and any other type of link. This approach is not inconsistent with the formalism that I will develop for showing the relationship between the inheritance links provided by Wille's concept lattices and the functions applied to the concepts created by Wille's concept lattices.

\section{Object-Oriented Solution}

While the concept lattice is an excellent way of deriving the inheritance hierarchy, a significant amount of expressiveness may be gained by switching to object-oriented notation. Figure 2 shows the concept lattice from Figure 1 represented using a Coad and Yourdon object-oriented analysis (OOA) diagram (Coad \& Yourdon, 1990). Notice that the inheritance hierarchy is completely preserved as the notation is switched from concept lattice to OOA notation. The concepts from the concept lattice are represented as classes in OOA, with the inheritance links represented as half circles. The classes themselves are divided into three sections. The first section of the class represents the name of the class. I have named the classes using their corresponding concept names from Figure 1. In the case of a concept having an object generator, I have additionally included the object name as part of the class name. The next two sections of the class represent the attributes and services of the class. The separation of attributes and services is a convenient way of distinguishing between nouns and verbs. Notice that the concepts B5 and B11 contain the same words in the attributes section as in the services section. This represents that the same word is a noun and a verb. Also notice that when the class represents a paragraph of R.I.T., all of the words are either attributes or services. This is because a single paragraph within R.I.T. contains only nouns or verbs.

Each of the services in the classes of an OOA diagram should have a defined behavior. Consider the verb toast in class B3. Class B3 inherits the service toast from class B11. Along with the inheritance links in Figure 2, I have also included the behavior for the verb toast. The OOA notation for a whole-part link is a small triangle. The OOA notation for a message is a thick lined arrow. The whole-part link may represent a uses relationship. In the case of the verb toast, the whole-part links represent that the action of toasting uses class B4 (containing toaster) and class B16 (containing bread). The message link has been used to show a constructor message sent to the B10 class (containing the noun toast). Similar links may be drawn for representing the behavior of the other services in class B3 and class B8.

\section{Type Theoretic Solution}

To understand better how the functions need to be represented in R.I.T., I will now examine how the classes and functions created using concept lattices and objectoriented diagramming may be represented in type theory (Cardelli \& Wegner, 1985). Using Cardelli and Wegner's idea of a type, each concept formed by the generation of the concept lattice is a type. The subtype relation defines a partial order on the set of all types and forms a lattice. Using Cardelli and Wegner's notation, I have translated the class B3 from Figure 2, along with the function defined, into a type representation. For the sake of brevity, I have omitted the function definitions that are not relevant for this example:

$$
\begin{aligned}
& \text { type } \mathrm{B} 3 \text { = } \\
& \text { \{toast: } \mathrm{B} 4 \times \mathrm{B} 16 \rightarrow \mathrm{B} 10, \\
& \text { bake: } \ldots, \\
& \text { baste: } \ldots, \\
& \ldots \text {. }
\end{aligned}
$$

Because each verb is listed as a separate service in class B3, each verb should have a separate function definition. Defining separate functions for each verb intuitively makes sense, because each verb will have different actions with different objects involved. The disturbing part of defining a separate function for each verb (as opposed to one function for each concept) is that there are so many verbs in R.I.T. 


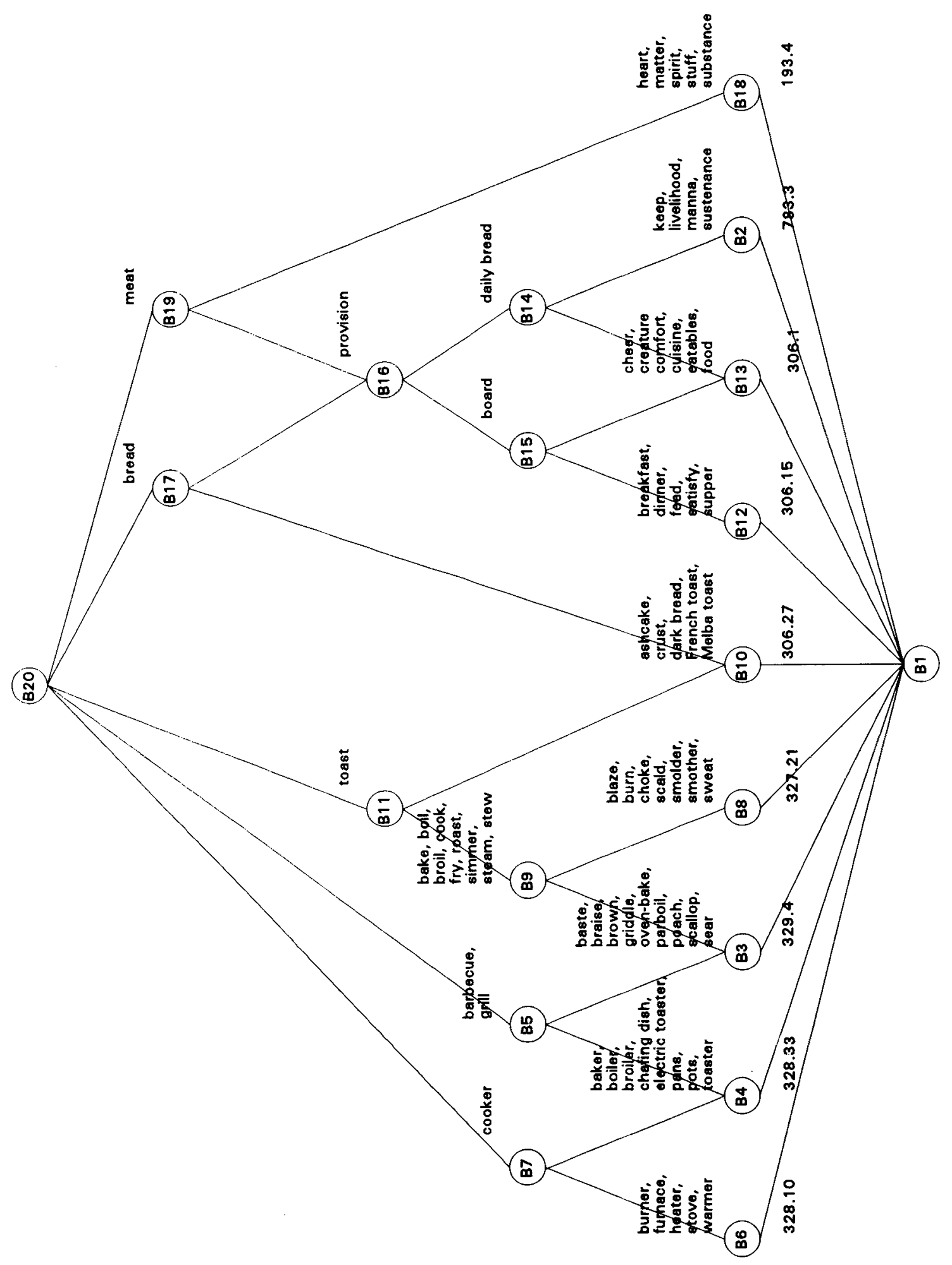




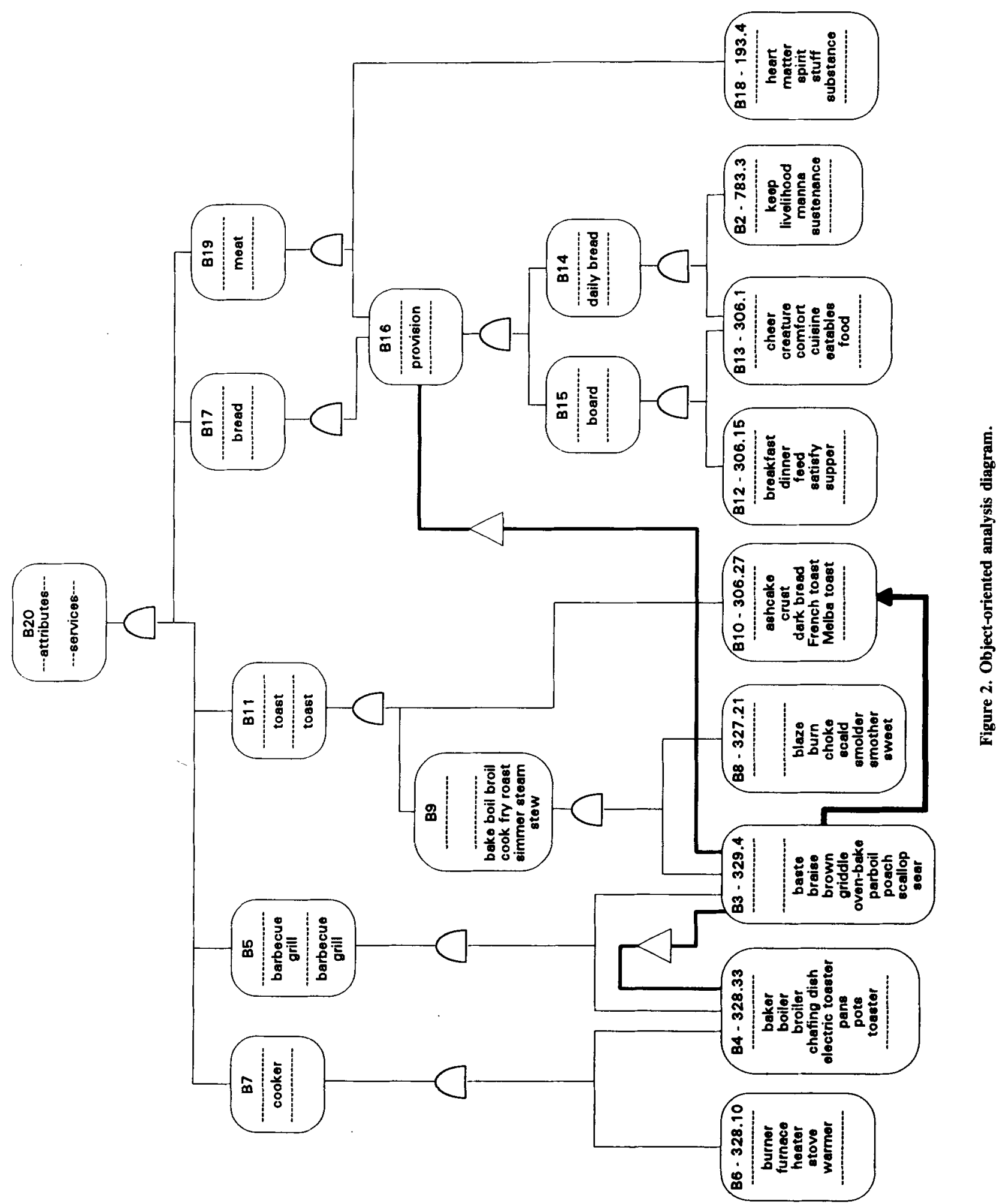


The verb toast is also present in the class B8 in Figure 2. This meaning of toast is not limited to toasting bread. Instead it refers to the idea of heating, or overheating, any substance. Since it will be interesting to compare classes $\mathrm{B} 3$ and $\mathrm{B} 8$, following is the type definition for B8:

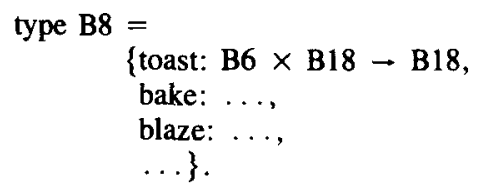

The definition for the toast function in type B8 takes a heater from B6, some substance from B18, and returns the same substance. Actually, the return value from the function should represent a hot substance. However, to simplify the lattice used in the example, I did not include any areas of R.I.T. dealing with hot substances.

Taking this example one step further, it will now be interesting to define the type B9. As can be seen from the inheritance hierarchy in Figure 1 or $2, \mathrm{~B} 9$ is the join of B3 and B8. Therefore, type B9 should be derivable as follows:

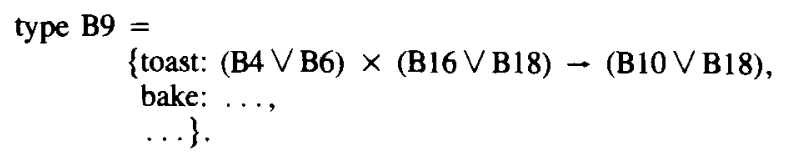

According to Cardelli and Wegner, as you follow a function from a subtype to its supertype, the domain of the function should expand, while the codomain of the function should shrink (Cardelli \& Wegner, 1985). If I were to follow this rule, the return value of the above function should specify the meet and read $(B 10 \wedge \mathrm{B} 18)$. However, it seems more intuitively correct when dealing with the English language to make all of the terms more generic.
Type B9 may be rewritten to resolve the joins:

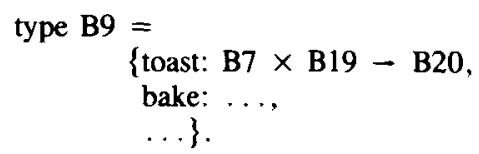

In fact, the concept lattice in Figure 1 contains the joins for the parameters of the toast function. However, the join for the return value is not present in the concept lattice below the top. It is also the case that the join of B10 and $\mathrm{B} 18$ does not exist in R.I.T. Perhaps this problem would be corrected if the toast function in type B8 were defined with a more appropriate return value than B18.

\section{Conclusion}

I have shown how Roget's International Thesaurus may be represented using concept lattices, object-orientation, and type theory. While some problems have been encountered formalizing the thesaurus, it is still very interesting and very valuable that formal techniques may be successfully applied to Roget's International Thesaurus. My future work will involve applying the notion of the object to the concept lattice in order to define behavior within the concepts.

\section{REFERENCES}

Cardelli, L., \& Wegner, P. (1985). On understanding types, data abstraction, and polymorphism. ACM Computing Surveys, 17, 471-522.

CoAD, P., \& Yourdon, E. (1990). Object-oriented analysis. Englewood Cliffs, NJ: Prentice-Hall.

Roget's International Thesaurus (1962). New York: Crowell, Thomas.

Thomason, R. H., and Toure tsky, D. S. (1991). Inheritance theory and networks with roles. In J. F. Sowa (Ed.), Principles of semantic networks: Explorations in the representation of knowledge (pp. 231266). San Mateo: Morgan Kaufmann.

WILLE, R. (1990). Concept lattices and conceptual knowledge systems. Computers \& Mathematics with Applications, 23, 493-515. 